The cranial ultrasound and the fundus eye were normal. Abdominal Doppler ultrasound was showed Compartmentalized cyst of the left kidney. The electroencephalography and the cardiac echography were normal.

Conclusion CMTC is a rare skin disease whose determinism remains unknown. Other congenital anomalies can be seen. The prognosis depends on the associated anomalies but it is generally good. Some cases have spontaneous resolution.

\section{P590 INVESTIGATING THE RELATIONSHIP BETWEEN PARENT DIABETES-RELATED DISTRESS AND FAMILY COMMUNICATION IN PARENTS OF ADOLESCENTS LIVING WITH TYPE 1 DIABETES}

${ }^{1}$ Caroline Rawdon* ${ }^{1}$ Pamela Gallagher, ${ }^{2}$ Michele Glacken, ${ }^{3,4}$ Nuala Murphy, ${ }^{5}$ Veronica Swallow, 'Veronica Lambert. 'Dublin City University, Dublin, Ireland; 'Institute of Technology, Sligo, Ireland; ${ }^{3}$ Temple Street Children's University Hospital, Dublin, Ireland; ${ }^{4}$ Mater Misericordiae University Hospital, Dublin, Ireland; ${ }^{5}$ University of Leeds, Leeds, UK

10.1136/archdischild-2019-epa.924

Aims Parents of adolescents living with type 1 diabetes often struggle with sharing self-management responsibility with their children as roles change throughout adolescence. Existing evidence suggests that parents' own emotions may impact how responsibility is shared. Parent diabetes-related distress may lead to ineffective communication between parents and adolescents throughout this developmental period as self-management moves from parent-led to adolescent-led. This study explored the relationship between parent diabetes-related distress, diabetes family responsibility, and parent-adolescent communication.

Methods Parents $(N=146 ; 121$ female; mean age $=46.56$ years) of adolescents (11-17 years) living with type 1 diabetes completed a questionnaire which included measures of parent diabetes-related distress, diabetes family responsibility, and parent-adolescent communication. Parents were recruited via one national paediatric diabetes and endocrine unit and a national diabetes advocacy organisation.

Findings Significant $(p<0.05)$ negative correlations were observed between parent diabetes distress and overall parentadolescent communication $(r=-0.570)$, as well as open communication $(r=-0.453)$ and problems in communication subscales $(r=-0.549)$. All subscales of the diabetes distress scale (personal distress, teen management distress, parent-teen relationship distress, and healthcare team distress) correlated negatively with overall parent-adolescent communication, open communication, and problems in communication. Most notably, parent-teen relationship distress correlated with overall parent-adolescent communication $(r=-0.679)$, open communication $(r=-0.527)$, and problems in communication $(r=-$ 0.664). A significant $(p<0.05)$ positive correlation was observed between parent diabetes distress and parental involvement in diabetes management $(r=0.357)$.

Conclusions Relationships between aspects of parent diabetesrelated distress and parent-adolescent communication were observed; higher levels of parent diabetes-related distress indicated less open communication and more problems in communication. Higher parent diabetes-related distress was associated with more parental involvement in adolescents' type 1 diabetes management. Adolescence is a key time in which diabetes management moves from parent-led to adolescent-led. Understanding the challenges that parents experience when sharing management responsibility and communicating with their adolescent children about type 1 diabetes management may help healthcare professional to provide more effective supports for parents and adolescents during this developmental period.

\section{P591 NEONATAL DIABETIS}

Amel Ben Hmed, Rim Zaghdoud, Chiraz Regaieg*, Manel Charfi, Amira Bouraoui, Ridha Regaieg, Nadia Hmida, Afzf Ben Thabet, Abdellatif Gargouri. Deparetement of Neonatology, CHU Hedi Chaker, Sfax, Tunisia

\subsection{6/archdischild-2019-epa.925}

Background Neonatal diabetes mellitus (NDM) is rare, noted in only 1 of every $300 \quad 000-400000$ live births. Both transient and permanent conditions have been described (TNDM and PNDM, respectively) which have different clinical, genetic features and outcomes.

Cases presentation Three newborns were included, 2 males and 1 female. The mean gestationnel age was: 35.3. All of them had born to consanguineous parents. The mean birth weight was $1506 \mathrm{~g}$. Two patients had a family history of neonatal hyperglycemia. By the first week of life, glucose concentrations increased, Ketonuria and glycosuria were noted in two cases, the third patient had hyperglycemia, glycosuria without ketonuria at the age of 2 months. The serum insulin and C-peptide levels were low. Tests for anti-islet cell and anti-glutamic acid decarboxylase antibodies were all negative. Abdominal ultrasound was normal. The infants were diagnosed with NDM. Genetic testing was performed. Results are not yet available. All patients were managed with insulin therapy (1-2.5 UI/kg/J). During follow up Recurrent hospital admission for diabetic ketoacidosis were noted in two cases, but long term outcome was favorable. One patient had died.

Conclusion Neonatal diabetes mellitus is uncommon. It is important to distinguish neonatal diabetes mellitus from other causes of hyperglycemia in newborns. Early recognition and urgent genetic testing are important for predicting the clinical course and raising awareness of possible additional features.

\section{P592 PAEDIATRIC ECG INTERPRETATION BY NON- CONSULTANT HOSPITAL DOCTORS (NCHDS) WORKING IN AN IRISH TERTIARY PAEDIATRIC CENTRE}

${ }^{1}$ Michael Pio Fitzgerald*, ${ }^{2}$ Catherine Crowe, ${ }^{3}$ Adam James, ${ }^{1}$ Patrick Fitzpatrick. ${ }^{1}$ Children's University Hospital, Temple Street, Dublin, Ireland; ${ }^{2}$ National University Ireland, Galway, Ireland; ${ }^{3}$ Our Lady's Children's Hospital Crumlin, Dublin, Ireland

\subsection{6/archdischild-2019-epa.926}

Introduction Paediatric ECG interpretation is a core clinical competence in postgraduate paediatric specialist training. Doctors treating paediatric patients with potential cardiac conditions should be familiar with the principles of ECG interpretation and the diagnoses requiring emergency treatment. Research has shown that paediatric ECG interpretation amongst doctors may be suboptimal and that the most effective method of teaching ECG interpretation is unclear. $^{1}$

Aims This study aims to establish the accuracy of paediatric ECG interpretation by NCHDs working in an Irish Tertiary Paediatric Hospital and to measure the impact of a teaching intervention.

Methodology NCHDs working in acute clinical areas in an Irish tertiary paediatric hospital were invited to participate $(n=45)$. Participants were asked to report three ECGs (long 